\title{
VIVÊNCIA DE PRAZER E SOFRIMENTO NAEQUIPE TÉCNICA EM ENFERMAGEM DO CENTRO DE TERAPIA INTENSIVA
}

Rafael Mondego Fontenele ${ }^{1}$ Célia Maria Santos Rezende ${ }^{2}$ Hariane Freitas Rocha Almeida ${ }^{3}$ Ana Patricia Fonseca Coelho Galvão ${ }^{3}$ Aline Sharlon Maciel Batista Ramos ${ }^{3}$ Cristina Maria Douat Loyola ${ }^{4}$
ORCID: https://orcid.org/0000-0002-8086-740X ORCID: https://orcid.org/0000-0002-5717-1578 ORCID: https://orcid.org/0000-0002-1685-7012 ORCID: http://orcid.org/0000-0003-3376-5678 ORCID: https://orcid.org/0000-0002-7648-2857 ORCID: https://orcid.org/0000-0003-2824-6531

Objetivo: conhecer a vivência de prazer e sofrimento na equipe técnica em enfermagem do centro de terapia intensiva. Método: pesquisa do tipo qualitativa com observação participante e sistemática e entrevistas realizadas com técnicos de enfermagem do CTI de um hospital em São Luís - MA. Foram entrevistados 26 técnicos de enfermagem com histórico de atestados médicos entre julho de 2016 e julho de 2017. Utilizou-se a análise de conteúdo para e hermenêutica-dialética para tratamento dos resultados. Resultados: verificou-se a vivência de prazer por trabalhar com pacientes graves, relacionando a profissão com atos de heroísmo. Contudo, identificou-se a vivência de sofrimento físico por usar o corpo como ferramenta de trabalho, e o sofrimento psíquico traduzido em rotinas carregadas de cobranças excessivas, e medo de perder o emprego levando à prática de presenteísmo. Conclusão: conclui-se que os técnicos de enfermagem do centro de terapia intensiva vivenciam prazer por sentimentos de heroísmo na profissão, e sofrimento relacionado ao trabalho devido a cobranças excessivas, vigilância permanente, problemas nas relações com a gestão de enfermagem, absenteísmo e sentimento de exclusão.

Descritores: Esgotamento Profissional; Saúde do Trabalhador; Profissionais de Enfermagem; Técnicos de Enfermagem; Enfermagem de Cuidados Críticos.

\section{PLEASURE AND SUFFERING LIVING IN THE TECHNICAL TEAM IN NURSING CENTER OF INTENSIVE THERAPY}

Objective: to know the experience of pleasure and suffering in the nursing team of the intensive care unit. Method: qualitative research with participant and systematic observation and interviews with nursing technicians from the ICU of a hospital in São Luís, Maranhão, Brazil. 26 nursing technicians with a history of medical certificates were interviewed between July 2016 and July 2017. Content analysis for and dialectic hermeneutics was used to treat the results. Results: the experience of pleasure was verified by working with serious patients, relating the profession with acts of heroism. However, the experience of physical suffering was identified by using the body as a work tool, and the psychic suffering translated into routines loaded with excessive charges, and fear of losing the job leading to the practice of presenteism. Conclusion: it is concluded that the nursing technicians of the intensive care center experience pleasure from feelings of heroism in the profession, and workrelated suffering due to excessive collections, permanent vigilance, problems with nursing management, absenteeism and feelings of exclusion. Descriptors: Burnout; Occupational Health; Nurse Practitioners; Licensed Practical Nurses; Critical Care Nursing.

\section{VIVENCIA DE PLACER Y SUFRIMIENTO EN EL EQUIPO TÉCNICA EN ENFERMERÍA DEL CENTRO DE TERAPIA INTENSIVA}

Objetivo: conocer la vivencia de placer y sufrimiento en el equipo técnico en enfermería del centro de terapia intensiva. Metodo: investigación del tipo cualitativo con observación participante y sistemática y entrevistas realizadas con técnicos de enfermería del CTI de un hospital en São Luís, Maranhão, Brasil. Se entrevistó a 26 técnicos de enfermería con historial de atestados médicos entre julio de 2016 y julio de 2017 . Se utilizó el análisis de contenido para y hermenéutica-dialéctica para tratamiento de los resultados. Resultados: se verificó la vivencia de placer por trabajar con pacientes graves, relacionando la profesión con actos de heroísmo. Sin embargo, se identificó la vivencia de sufrimiento físico por usar el cuerpo como herramienta de trabajo, y el sufrimiento psíquico traducido en rutinas cargadas de cobranzas excesivas, y miedo de perder el empleo llevando a la práctica de presenteísmo. Conclusión: se concluye que los técnicos de enfermería del centro de terapia intensiva experimentan placer por sentimientos de heroísmo en la profesión, y sufrimiento relacionado al trabajo debido a cargos excesivos, vigilancia permanente, problemas en las relaciones con la gestión de enfermería, absentismo y sentimiento la exclusión.

Descriptores: Agotamiento Profesional; Salud Laboral; Enfermeras Practicantes; Enfermeros no Diplomados; Enfermería de Cuidados Críticos.

${ }^{1}$ Instituto de Ensino Superior Franciscano (IESF-MA)

${ }^{2}$ Faculdade Gianna Beretta

${ }^{3}$ Universidade Ceuma, MA

${ }^{4}$ Universidade Ceuma e Universidade Federal do Rio de Janeiro

Autor correspondente: Rafael Mondego Fontenele E-mail: fhaelmondego@gmail.com

Recebido: 19/11/2018

Aceito: 23/09/2019 


\section{INTRODUÇÃO}

O trabalho da equipe de enfermagem na Unidade de Terapia Intensiva (UTI) necessita de forte envolvimento afetivo em virtude da necessidade de vigilância constante que se faz necessária para a produção do cuidado holístico proposto pela enfermagem como ciência. Esta visão de trabalho holístico produz inúmeras outras atribuições para a equipe de enfermagem, contudo, não deve ser um fator dificultador para a assistência destes profissionais ${ }^{(1)}$.

Considerando a gravidade dos doentes internados em uma UTI, cabe à enfermagem garantir a segurança dos pacientes, pois se trata de um compromisso ético da formação. Sendo assim, se este pré-requisito não é atendido, a qualidade da assistência de enfermagem pode ser prejudicada, afetando - cuidado necessário para a recuperação dos doentes ${ }^{(2)}$. Para a preservação destes fundamentos, é necessária a concentração de esforços dos membros da equipe de enfermagem, fato que pode estar levando estes trabalhadores a vivenciar prazer e sofrimento relacionado ao trabalho.

Sobre o risco de adoecimento dos profissionais da equipe de enfermagem, o prazer e o sofrimento relacionado ao trabalho ganham destaque, como descrito na psicodinâmica do trabalho em que há um misto de prazer e sofrimento, este, quando maior que o prazer, pode levar os trabalhadores dessa categoria profissional a graves problemas de saúde ${ }^{(3)}$.

Sobre a vivência de prazer e sofrimento pela equipe de enfermagem, vários fatores influenciam esses sentimentos, os quais estão vinculados à relação interpessoal entre pacientes, ou à perda dos mesmos, o que pode traduzir sentimento de frustração, além do estresse da dificuldade de inserir as famílias dos pacientes no contexto, impossibilidade de crescimento, sobrecarga de trabalho, jornada extensa e baixo salário(4).

A justificativa para este estudo baseia-se na necessidade de conhecer a vivência de prazer e sofrimento para que se possa implementar continuamente as medidas de prevenção e promoção à saúde dos profissionais de enfermagem, tendo em vista que estes profissionais estão em constantes situações de estresse, condições precárias de trabalho ou baixas remunerações, e pouco ou nenhum reconhecimento profissional que viabilizam o sofrimento psíquico predispondo os cuidados inseguros que podem comprometer a qualidade da assistência de enfermagem ${ }^{(5)}$.

Sendo assim, considerando os problemas que se produzem quando as relações não são fortalecidas com o intuito de gerar prazer em relação ao trabalho, o objetivo do presente estudo foi conhecer a vivência de prazer e sofrimento na equipe técnica em enfermagem de um centro de terapia intensiva.

\section{METODOLOGIA}

\section{Tipo de estudo}

Trata-se de pesquisa qualitativa, descritiva, com observação participante e observação sistemática, entrevistas semiestruturadas e análise documental.

\section{Participantes da pesquisa}

Foram entrevistados os técnicos de enfermagem que trabalhavam no CTI, e utilizou-se o método de saturação teórica em pesquisa qualitativa(b) ${ }^{(6)}$ como critério para finalização de obtenção de dados empíricos. Foram incluídos no estudo os técnicos de enfermagem de ambos os sexos que apresentaram pelo menos um afastamento de suas atividades por motivo de doença, ao qual apresentou justificativa da falta ao trabalho por meio de atestado médico no período de julho de 2016 a julho de 2017. Foram excluídos do estudo todos os profissionais que estavam em gozo de férias, licença por doença, licença maternidade ou paternidade no período da coleta de dados. A amostra final foi constituída por 26 técnicos de enfermagem.

\section{Local do estudo}

A presente pesquisa foi realizada em um Centro de Terapia Intensiva de um hospital privado no município de São Luís no Estado do Maranhão.

\section{Coleta de dados}

Os dados foram coletados no período de agosto de 2017 a janeiro de 2018. Inicialmente, realizou-se levantamento documental junto ao serviço de medicina do trabalho, que procedeu à emissão do relatório de técnicos de enfermagem com histórico de pelo menos um afastamento por motivo de doença no período de julho de 2016 a julho de 2017. Em seguida, foi iniciada a observação participante e sistemática com registro em diário de campo.

A observação participante acompanhou os profissionais durante a rotina de plantões nos três turnos. Neste método, o pesquisador participa e interfere no contexto investigado, com ênfase nas ações dos participantes e não no ambiente propriamente dito(7).

Para a realização da observação sistemática, utilizou-se um checklist ${ }^{(8)}$ contendo itens de observação, neste estudo, adotados como: aspectos e fatores que produzem prazer ou sofrimento, ambos relacionados ao trabalho, e cujo registro foi realizado em diário de campo, a fim de compreender as interpretações do grupo acerca dos acontecimentos.

Ambas as técnicas de observação foram realizadas nos três turnos no período de 27 de agosto de 2017 a 16 de outubro de 2017, antes que fosse iniciada a etapa de entrevistas 
individuais para garantir a invisibilidade do pesquisador e distanciar respostas pré fixadas pelos participantes da pesquisa, e totalizaram 253 horas de registro em diário de campo. As observações permitiram identificar os potenciais participantes para o presente estudo.

As entrevistas individuais foram realizadas em um ambiente fora do hospital para diminuir possivel constrangimento dos depoentes, gravadas, conforme o método de pesquisa qualitativa, e em seguidas transcritas pelo próprio pesquisador, entre o período de 7 de novembro de 2017 a 23 de janeiro de 2018. O local da entrevista foi definido pelo entrevistado, e cada entrevista durou, em média, 25 minutos. As falas dos participantes foram identificadas pela letra "T", os fragmentos das anotações do diário de campo foram identificados no corpo do texto seguidos de "DC".

\section{Procedimentos de análise dos dados}

A análise do material empírico foi realizada a partir da triangulação dos dados obtidos pela observação participante e sistemática, entrevistas e referencial teórico. Para análise do material empírico, utilizou-se a técnica de análise temática, através da pré-análise, que consistiu na leitura dos textos das entrevistas transcritas, para a retomada de hipóteses e dos objetivos iniciais da pesquisa, reformulando-as frente ao material coletado e na elaboração de indicadores que orientem a interpretação final. Nesta etapa, a leitura flutuante permitiu a escolha das entrevistas que seriam utilizadas, com base na definição dos núcleos de sentido. Em seguida, realizou-se a categorização, a partir da exploração do material ou codificação dos dados, que se caracteriza pela transformação dos dados brutos, visando a alcançar o núcleo de compreensão do texto. Nesta etapa, agruparam-se os núcleos de sentido com a construção de categorias para análise. E por fim, a interpretação através do tratamento dos resultados obtidos, baseando-se na proposição de inferências e interpretações previstas no quadro teórico(9).

Os dados foram analisados à luz da hermenêutica dialética, a partir da subjetivação do objeto e da objetivação do sujeito ${ }^{(10)}$.

\section{Procedimentos éticos}

O presente estudo foi submetido à Plataforma Brasil e obteve aprovação do Comitê de Ética em Pesquisa com CAAE no 67664217.6.0000.5085, sob o parecer n우 2.234.735. A coleta de dados foi iniciada após explicação dos processos éticos que envolvem o estudo, baseado em regulamentações pertinentes, bem como a assinatura do termo de consentimento livre e esclarecido por ambas as partes. Este artigo foi extraido de uma dissertação de mestrado com o recorte de duas categorias operacionais.

\section{RESULTADOS}

\section{Caracterização dos participantes da pesquisa}

A maioria dos participantes do estudo foram mulheres jovens, com idade entre 24 e 49 anos, casadas ou solteiras, com ensino superior completo ou graduação em curso, entre 1 a 3 vínculos de emprego, perfazendo renda individual entre $\mathrm{R} \$ 1.201,00$ e $\mathrm{R} \$ 2.500,00$ por mês.

\section{Sentimentos de prazer por trabalhar na UTI}

O prazer repercutiu, pois a maioria desses profissionais gosta do trabalho na UTI. O gostar geralmente estava relacionado com a existência de equipamentos necessários ao cuidado de enfermagem e cuja falta produz ansiedade e expõe o paciente ao risco, como destaca a fala a seguir:

"Gosto. Por causa dos recursos que são disponibilizados aqui para todos nós, principalmente para prevenção" (TO4).

O trabalho na UTI remete a sentimentos de heroísmo, como destacou o profissional, em um discurso de zelo:

"Eu amo trabalhar na UTI, na correria, na adrenalina, salvando vida, recebendo o paciente que "não vai nem amanhecer' e passar o plantão com o paciente vivo" (TO5).

A gestão de enfermagem compreende as faltas por motivo de doença. Esta compreensão produz admiração e exacerbação da competência desses gestores, na perspectiva dos entrevistados, porém com contradições:

“Sempre foi uma relação de muita compreensão. É só avisar que você não vai poder vir. Elas não gostam de ser pegas de surpresa" (TO6),

"Mesmo quando avisamos que estamos doentes e que vamos faltar, elas não gostam" (TO3).

\section{Vivência de sofrimento na UTI}

\subsection{Cobranças excessivas de normas e rotinas}

A insatisfação pelo trabalho aparece em questionamentos sobre normas e rotinas:

“É chato. Cheio de regras. Se você acerta, não tem elogios. Mas se você vacila, é chamada a atenção" (TO2).

Destacaram-se cobranças excessivas, sobretudo, quanto à mudança de decúbito em aprazamentos que ocorrem de duas em duas horas. Há uma desproporção entre as estaturas dos clientes a serem mobilizados e a estatura dos profissionais de saúde. Há um consenso da ligação desta realidade com problemas de coluna e afastamentos do serviço: 
"É bom para o paciente, mas prejudica o funcionário [...] tem gente lá que só vive com problemas na coluna" (T18).

Para os participantes do estudo, ter que mudar o decúbito do paciente a cada duas horas é um fator que predispõe o estresse, qualificando esta atribuição como penosa e dolorosa.

\subsection{Vigilância permanente}

A existência de uma câmara de filmagem, criando um ambiente de vigilância permanente onde nada pode escapar aos coordenadores e supervisores, tem produzido ansiedade e insatisfação, como descrita no discurso:

"A gente trabalha debaixo dessas câmeras com a sensação de estar sendo vigiado o tempo inteiro" (TO8).

\subsection{Sofrimento traduzido em absenteísmo}

O absenteísmo produz estresse na equipe de técnicos de enfermagem. E quando as faltas são frequentes, é preciso explicar. Explicar a doença pode estar produzindo sofrimento psíquico nos trabalhadores:

"Um dia eu avisei que meu filho estava internado e nesse dia me questionaram 'de novo atestado?'. Isso faz a gente ficar um pouco abalada emocionalmente" (TIO).

\subsection{Sofrimento pelas relações com a gestão de enfermagem}

Há problemas interpessoais na relação entre os enfermeiros supervisores e técnicos em enfermagem:

"Eu tenho uns conflitos com uma supervisora especifica. Faz comentários, trata as pessoas sem educação" (T15).

Destacam-se relatos de desconfortos nos profissionais que se sentem, por vezes, expostos:

“Tem uns relatórios dos supervisores que indicamos nomes das pessoas e eu acho uma exposição desnecessária. Isso me deixa insatisfeita e com medo de perder o emprego" (TI6).

Este modelo de gestão dos recursos humanos tem produzido insatisfação e sentimento de revolta:

"Teve um tempo que eu me desentendia com supervisor. Agora eu os deixo falando sozinho" (TI8).

Há contradições quanto a comunicar faltas com antecedência:
"Eles não estão preocupados não. Se você está doente, você tem que ir trabalhar, você tem que se virar" (TO2). Outro profissional destacou: "Entendem na maioria das vezes como desculpa esfarrapada" (TO4).

Observou-se uma gestão focada em resultados e que as cobranças pelo alcance de metas e estatísticas produzem ansiedade nos trabalhadores:

"Sinto que fui contratado para fazer meu trabalho e ir embora, ninguém se preocupa com minha doença" (TO3).

\subsection{Sofrimento pelo sentimento de exclusão}

Os discursos dos participantes apresentam, por vezes, sentimentos de "serem excluídos" dos demais membros da equipe pelo fato de serem reconhecidos apenas como "executores de tarefas" dentro do contexto hospitalar:

"Quando tem aniversário, lanche, pizza, essas coisas aqui no hospital, os técnicos não são convidados" (TO1).

A segregação de niveis, entre técnico e superior, é real e deixa o técnico em enfermagem sempre na condição de subordinação, pois sempre estará submisso às ordens dos demais níveis acadêmicos, acentuando seu afastamento da equipe.

A relação de trabalho entre enfermeiros e técnicos de enfermagem parece sem significação para "equipe de enfermagem", pois o técnico sente-se excluído dessa equipe:

“Parece que há uma separação de niveis que é um absurdo de existir dentro da própria equipe de enfermagem. Por exemplo, o enfermeiro não se mistura com os técnicos porque parece que a gente suja eles" (TOI).

\section{DISCUSSÃO}

No presente estudo, o prazer pelo trabalho está destacado por sentimentos de heroísmo, como salvar vidas e com a disponibilização de recursos materiais que tornam a assistência de enfermagem segura e motiva a dedicação da equipe de enfermagem ao paciente grave. A partir desta perspectiva, e ao correlacionar os achados da presente pesquisa com sentimentos de prazer destacados por profissionais da terapia intensiva (11), é possivel refletir que o prazer pelo trabalho é incentivado pela motivação para o trabalho.

Em contrapartida, o excesso de normas e cobranças, torna o trabalho mais burocrático e também produz insatisfação para o mesmo, corroborando com achados da presente pesquisa ${ }^{(12)}$. 
Ao estudaras vivências deprazer esofrimento em uma equipe de profissionais de saúde em Mato Grosso do Sul, foi possivel reforçar que a ausência de insumos, ou a demora na aquisição destes, produz descontentamento no profissional(13). Desta forma, a precariedade do sistema de saúde com baixa oferta de serviços ou de materiais para realização de procedimentos também pode influenciar na relação de experiência de prazer e sofrimento.

Em relação ao esforço físico, técnicos e auxiliares de enfermagem de um hospital de Porto Alegre, destacaram que existe uma demanda de esforço físico inerente ao trabalho da enfermagem, principalmente em pacientes acamados, produzindo distúrbios osteomusculares nos trabalhadores ${ }^{(14)}$. Diante deste fato, vale ressaltar que os serviços de saúde precisam empregar maiores possibilidades para a execução das tarefas fundamentais da assistência de enfermagem, minimizando o esforço da equipe de enfermagem em procedimentos rotineiros na UTI, como a mudança de decúbito devido ao risco de integridade da pele prejudicada.

Os distúrbios osteomusculares atingem mais diretamente os auxiliares e técnicos de enfermagem do que os enfermeiros, pois geralmente o enfermeiro assume papéis administrativos que o distancia da assistência ao paciente ${ }^{(15)}$.

Sobre o sentimento de estar vigiado constantemente, pesquisa destacou que a vigilância por vídeo no trabalho tem representado a perda da privacidade do trabalhador, aumentado o índice de insatisfação no trabalho(16). Por outro lado, a existência de câmeras de vigilância torna-se importante aliada no que diz respeito a segurança do paciente ao possibilitar a comprovação da realização do cuidado pela equipe multiprofissional.

Sobre o absenteísmo, a compreensão das faltas da equipe é um ponto positivo para os chefes, corroborando os discursos dos entrevistados no presente estudo que a partir desta compreensão, entendem que a relação com a gestão tornase flexivel(17). Contudo, a flexibilização de atrasos e faltas pode comprometer a qualidade da assistência de enfermagem.

Sobre as ausências e flexibilização de faltas e atrasos, um hospital universitário brasileiro destacou o comprometimento da qualidade da assistência prestada ${ }^{(18)}$. Deve-se considerarainda que as ausências no plantão têm potencial de desestruturar toda a dinâmica da equipe pelo fato de sobrecarregar as atividades dos demais trabalhadores e favorecer a produção de estresse e sofrimento.

Pesquisa sobre gestão da enfermagem, destacou que a interação entre gestores e membros da equipe com o meio ambiente de trabalho facilitaram a gerência ${ }^{(19)}$. Contudo, as relações interpessoais e a falta de capacitação dos gestores e supervisores foram apontadas como formas de gerar insatisfação para o trabalho(20).

O medo de perder o emprego também foi descrito em estudo que corrobora com os achados desta pesquisa ${ }^{(21)}$.
Cabe ressaltar que o medo de perder o emprego caracteriza sofrimento para o trabalhador e predispõe a problemas de saúde de ordem psíquica, quando o trabalhador se submete às pressões, ameaças ou exigências como forma de garantir o trabalho.

Sobre o processo de trabalho da equipe de enfermagem, técnicos de enfermagem relataram o sentimento de exclusão da equipe multiprofissional, sentindo-se por vezes apenas como executor de tarefas, corroborando com as falas dos participantes da presente pesquisa(22).

A pressão por resultados está entre os principais fatores que produzem sofrimento relacionado ao trabalho, pois o fracasso no alcance das metas estipuladas está intimamente conectado a uma demissão imediata(23), este fenômeno evidencia-se, sobretudo, quando a gestão do trabalhador está sob a responsabilidade de lideranças centralizadoras que podem contribuir para o adoecimento psíquico.

\section{Limitações do estudo}

A limitação deste estudo refere-se às múltiplas faces do sofrimento psíquico para o trabalhador de enfermagem, pois a tensão laboral pode estar relacionada a fatores físicos, químicos, biológicos, sociais e/ou econômicos, que exercem efeitos geralmente a partir de ações simultâneas e interações entre tais fatores que dificulta a vivência de prazer relacionado ao trabalho.

\section{Contribuições para a prática}

Esta pesquisa serve de alerta para locais com condições insalubres e poderá contribuir para o enriquecimento das discussões acerca da implementação de medidas de rastreamento e diminuição do sofrimento relacionado ao trabalho da equipe de enfermagem, favorecendo a saúde mental destes trabalhadores.

\section{CONCLUSÕES}

O prazer pelo trabalho na UTI está relacionado a gostar do que se faz e, por consequência, ao fato de salvar vidas como evento desencadeador de sentimentos de heroísmo.

A existência de câmeras de vídeo produz ansiedade e prejudica a individualidade do profissional, tornando a assistência mecanicista. Há foco na segurança do paciente, mas deve-se implementar medidas que possam contribuir para a qualidade de vida e prazer pelo trabalho para os técnicos de enfermagem, devolvendo sua individualidade e privacidade.

Concluiu-se, ainda, que o excesso de normas e exigências tenha produzido sofrimento. Os depoentes apontam a utilização do corpo como ferramenta de trabalho responsável por dores físicas e sofrimento no trabalho, o que reflete no absenteísmo e sugere maior investigação.

Ademais, os problemas interpessoais e o sentimento de 
exclusão entre a equipe de enfermagem também configuram fontes geradoras de sofrimento no trabalho, podendo, consequentemente, elevar as taxas de absenteísmo no hospital.

Portanto, sugerem-se a adoção de estratégias como ginástica laboral regular, além de treinamentos com a equipe multiprofissional e lideranças, de modo a melhorar os vínculos entre as equipes, prevenindo ações e atitudes que possam estar produzindo sofrimento psíquico nestes trabalhadores.

\section{REFERÊNCIAS}

1. Ferreira MJM, Dodt RCM, Lima AM, Marques DRF, Pinheiro SMPR. Percepção dos acompanhantes sobre dispositivos invasivos em uma unidade de terapia intensiva. Enferm Foco [Internet].2018 [Cited 2017 16]: 9 (2): 18-22. Availabe from: http://revista.cofen.gov.br/index.php/enfermagem/article/view/1031.

2. Silva RC, Ferreira MA, Apostolidis T, Sauthier M. Práticas de cuidado de enfermagem na terapia intensiva: análise segundo a ética da responsabilidade. Esc Anna Nery [Internet]. 2016 [cited 2018 Nov 10]:20(4):e20160095. Available from: www.scielo.br/pdf/ean/v20n4/ 1414-8145-ean-20-04-20160095.pdf.

3. Worm FAA, Pinto MAAO, Schiavenato D, Ascari RA, Trindade LL, Silva OM. Risco de adoecimento dos profissionais de enfermagem no trabalho em atendimento móvel de urgência. Rev Cuid [Internet]. 2016 [cited 2018 Nov 9]:7(2):1279-1287 . Available from: www.scielo.org.co/scielo. php?script=sci_arttext\&pid=\$2216-09732016000200006\&lang=pt.

4. Caram CS, Rezende LC, Montenegro LC, Amaral JM, Brito MJM. Ambiguidades no trabalho da equipe de saúde no contexto de uma unidade de terapia intensiva. Sanare. [Internet]. 2016 [cited 2019 Jan 13]; 15(1):15-24. Available from: https://sanare.emnuvens.com.br/sanare/ article/view/923.

5. Lira MAM. Os efeitos na saúde das condições de trabalho da equipe de enfermagem da estratégia saúde da familia: uma revisão de literatura. Multi Scient Journal [Internet]. 2017 [cited 2019 Jan 13]; 9(3):5162. Available from: https://www.nucleodoconhecimento.com.br/saude/ saude-trabalho.

6. Fontanella BJB, Ricas J, Turato ER. Amostragem por saturação em pesquisas qualitativas em saúde: contribuições teóricas. Cad. Saúde Pública [Internet]. 2008 [cited 2018 Jan 20]; 24(1):17-27. Available from: http://www.scielo.br/pdf/csp/v24nl/02.pdf

7. Marietto ML. Observação participante e não participante: contextualização teórica e sugestão de roteiro para aplicação dos métodos. Iberoamerican Journal Of Strategic Management (IJSM) [Internet] 2018 [cited 2018 Jan 20]; 17(4): 05-18. Available from: http://dx.doi. org/10.5585/ijsm.v17i4.2717.

8. Torres GMC, Figueiredo IDT, Cândido JAB, Almeida MI. O emprego das tecnologias leves no cuidado ao hipertenso na Estratégia Saúde da Familia. Esc. Anna Nery [Internet]. 2018 [cited 2018 Jan 20]; 22(3): 1-8. Available from: http://dx.doi.org/10.1590/2177-9465-ean-2017-0169.

9. Minayo MCS. O desafio do Conhecimento: pesquisa qualitativa em saúde./ Maria Cecilia de Sousa Minayo. - 14. Ed. - São Paulo: Hucitec. 2014. $407 p$

10. Cardoso MF, Batista-dos-Santos AC, Alloufa JML. Sujeito, linguagem, idologia, mundo: técnica hermenêutico-dialética para análise de dados qualitativos de estudos críticos em administração. R. Adm. FACES Journal [Internet]. 2015 [cited 2018 Jan 20]; 14(2):74-93 Available from: http://dx.doi.org/10.21714/1984-6975FACES2015V14N2ART2112.

11. Padilha KG, Barbosa RL, Andolhe R, Oliveira EM, Ducci AJ, Bregalda RS et al. Carga de trabalho de enfermagem, estresse/Burnout, satisfação e incidentes em unidade de terapia intensiva de trauma. Texto Contexto Enferm [Internet]. 2017 [cited 2018 Jan 24]; 26(3):1-8. Available from: http://www.scielo.br/pdf/tce/v26n3/0104-0707-tce-2603-el720016.pdf.

12. Soratto J, Pires DEP, Trindade LL, Oliveira JSA, Forte ECN, Melo TP. Insatisfação no trabalho de profissionais da saúde na estratégia da saúde da familia. Texto Contexto Enferm [Internet]. 2017 [cited
2018 Jan 20]: 26(3):1-11. Available from: http://www.scielo.br/pdf/tce/ v26n3/0104-0707-tce-26-03-e2500016.pdf.

13. Azevedo APF, Figueiredo VCN. Vivências de prazer e sofrimento mental em um Centro de Atenção Psicossocial. Rev. Psicol., Organ. Trab., [Internet]. 2015 [cited 2018 Jan 23]; 15(1):30-42. Available from: http://pepsic.bvsalud.org/pdf/rpot/v15nl/v15nlaO4.pdf

14. Möller G, Magalhães AMM. Banho no leito: carga de trabalho da equipe de enfermagem e segurança do paciente. Texto Contexto Enferm [Internet]. 2015 [cited 2018 Jan 24]; 24(4): 1044-52. Available from: http://www.scielo.br/pdf/tce/v24n4/pt_0104-0707-tce-24-04-01044.pdf.

15. Portela NLC. Ross JR. Distúrbios osteomusculares relacionados ao trabalho (DORT) e sua associação com condições de trabalho da enfermagem. Rev Enferm UFPI [Internet]. 2015 [cited 2018 Jan 20];4(4):82-87. Available from: http://www.ojs.ufpi.br/index.php/reufpi/ article/view/2754/pdf

16. SoutoYF, Araújo JM. Direito à privacidade do trabalhador e o uso do correio eletrônico: violação de direitos fundamentais ou legítimo exercicio do poder direito do empregador? Revista Eletrônica da Escola Judicial do TRT 13a Região [Internet]. 2016 [cited 2018 Jan 27]; 1(1):210-232. Available from: http://www.egov.ufsc.br/portal/sites/default/files/direito_a_privacidade_do_trabalhador_e_o_uso_do_correio_eletronico_violacao_de_direitos_fundamentais_ou_legitimo_ exercicio_do_poder_diretivo_do_empregador.pdf.

17. Kurcgant P, Passos AR, Oliveira JML, Pereira IM, Costa TF. Absenteismo do pessoal de enfermagem: decisões e acões de enfermeiros gerentes. Rev Esc Enferm USP [Internet]. 2015 [cited 2018 Jan 24]; 49(Esp2):35-41. Available from: http://dx.doi.org/10.1590/S0080623420150000800005

18. Marques DO, Pereira MS, Souza ACS, Vila VSC, Almeida CCOF, Oliveira EC. Absenteeism - ilness of the nursing staff of a university hospital. Rev Bras Enferm [Internet]. 2015 [cited 2018 Jan 25]; 68(5):594600. Availablefrom: http://dx.doi.org/10.1590/0034-7167.2015680516i.

19. Rabenschlag LA, Lima SBS, Eberthardt TD, Kessler M, Soares RSA, Camponogara S. Gestão da qualidade na assistência de enfermagem em unidades de clínica cirúrgica. Rev Enferm USFM [Internet]. 2015 [cited 2018 Jan 25]; 5(2): 235-246. Available from: http://dx.doi. org/10.5902/2179769215891.

20. Fortes ECN, Pires DEP. Enfermeiras na atenção básica: entre a satisfação e a insatisfação no trabalho. Trab. Educ. Saúde [Internet]. 2017 [cited 2018 Jan 24]; 15(3):709-724. Available from: http://dx.doi. org/10.1590/1981-7746-sol00083.

21. Batista MA, Lima MEA, Antipoff RBF. A preservação da saúde em situações patogênicas de trabalho: um estudo de caso na siderurgia. Cadernos de Psicologia Social do Trabalho [Internet]. 2016 [cited 2018 Jan 20]:19(1):45-59. Available from: http://www.revistas.usp.br/cpst/ article/view/125901/122755

22. Samudio AKM, Loureiro MDR, Ferreira Júnior MAF. O processo de trabalho da equipe de enfermagem em cuidados continuados integrados. Rev enferm UFPE [Internet]. 2016 [cited 2018 Jan 24];10(7):245362. Available from: https://periodicos.ufpe.br/revistas/revistaenfermagem/article/viewFile/11302/12970.

23. Máximo TACO, Araújo AJS, Zambroni-de-Souza PC. Vivências de sofrimento e prazer no trabalho de gerentes de banco. Psicol. Cienc. Prof. [Internet]. 2014 [cited 2018 Jan 20]; 34(1):96-111. Available from: http://dx.doi.org/10.1590/S1414-98932014000100008. 